\title{
Efficiency Defence, Administrative Fuzziness, and Commitment in Merger Regulation
}

by

\author{
Andrei Medvedev \\ ESRC Centre for Competition Policy, University of East Anglia \\ CCP Working Paper 06-8
}

\begin{abstract}
This paper develops a signalling model to look at some effects of the inclusion of an efficiency defence in merger regulation. By incorporating Type I and Type II errors into the antitrust agency's pay-off function approval probabilities are endogenized. The agency can choose to use a fuzzy approval rule (mixed strategies) after observing a double signal (produced evidence and the way it has been produced) as a tool to (partially) separate different merger types by changing approval probabilities and, consequently, firms' expected payoffs from a merger. The separation leads to a lower value of the expected mistake by the agency. If the agency can commit to certain policies, then a fuzzy approval rule is preferred under a wide range of parameters.
\end{abstract}

May 2006

JEL Classification: K21; L44; L51

Keywords: Merger Regulation, Efficiency Defence, Signalling, Commitment

Acknowledgements:

I am thankful to Avner Shaked, Dirk Engelmann, Jan Boone, Bruce Lyons, Eric van Damme, Randall Filer, and Emiliya Lazarova for valuable discussions and suggestions. The support of the Economic and Social Research Council (UK) is gratefully acknowledged.

Contact details:

a.medvedev@uea.ac.uk, ESRC Centre for Competition Policy, University of East Anglia, Norwich, NR4 7TJ, UK. www.ccp.uea.ac.uk t: +44 (0) 1603593715 f: + 44(0) 1603 591622

ISSN 1745-9648 


\section{Introduction}

Recently, many countries have allowed for an efficiency defense in horizontal merger regulation. ${ }^{1}$ The efficiency defense refers to a case in which an antitrust agency is ready to approve a merger if a substantial increase in market concentration is 'compensated' by efficiencies caused by the merger. To be considered the efficiencies should be verifiable, merger specific, and passed on to consumers, i.e. to decrease prices. $^{2}$ However, the inclusion of the efficiency defense into merger regulation creates an asymmetric information problem between an antitrust agency and merging firms concerning the actual cost efficiencies due to the merger.

There are administrative and adversarial systems regarding merger regulation. The administrative system exists when the agency by itself estimates actual cost efficiencies caused by the merger and, based on this estimation, makes a decision to approve or reject a merger (in the EU). The adversarial system exists when the burden of proof to verify required cost efficiencies lies with the merging firms (in the USA and Canada). The adversarial system provides an opportunity for both 'good' and 'bad' mergers to take some action in order to improve their chances of getting approval. In this paper, I will analyze the adversarial system with the possibility of signaling.

There is an extensive literature on mergers for different market structures. ${ }^{3}$ If there are no cost reductions due to a merger, firms find it profitable to exercise their market power through price increases. However, if a merger would lead to significant synergies (efficiencies) between merging firms, then it could lead to a decrease in price. $^{4}$ Actual efficiencies, however, are not observable by the agency but it is assumed that merging firms know the true value. Merging firms can strategically reveal information or even cheat in order to get approval: "no class of evidence is free of the possibility of fabrication". ${ }^{5}$ All mergers have a positive probability of producing sufficient evidence at a resource expense. ${ }^{6}$ A merging firm chooses an effort level to produce verifiable evidence that efficiencies are sufficient to satisfy the relevant

\footnotetext{
${ }^{1}$ US, EU, Canada, UK, France, and Sweden consider the efficiency defense in their decisions.

${ }^{2}$ Yao and Dahdouh (1993); EU and US Horizontal Merger Guidelines.

${ }^{3}$ Salant, Switzer, and Reynolds (1983); Perry and Porter (1985); Deneckere and Davidson (1985); Zang and Kamien (1990); and Farrell and Shapiro (1990).

${ }^{4}$ See Williamson (1968); Werden (1996); Farrell and Shapiro (1990); Roller, Stennek, and Verboven (2000); Besanko and Spulber (1993); and Medvedev (2004a).

${ }^{5}$ U.S. Federal Rules of Evidence, rule 801(a), advisory committee's note.

${ }^{6}$ See Sanchirico (2001), where the evidence production process is described. It is assumed that the evidence can be produced or not ( 0 or 1$)$ rather than a continuous outcome between zero and one.
} 
welfare standard. ${ }^{7}$ The agency can observe the effort level (eg. Nobel Prize winners in expert witness testimonies, quality of lawyers and consulting firms). Therefore, an observable effort level to produce evidence is valuable information and neglecting it would not be optimal behavior by the agency. Empirically, some competition practitioners report that they do not care how the evidence was produced nor who produced and presented it - a Nobel Prize winner or an ordinary economist. ${ }^{8}$ Their only concern is the evidence itself. Why not treat the firms' efforts to produce the evidence as a signal?

In this paper, a new modification of the signaling game is constructed. In the model the effort level and type of merger determine the probability of producing verifiable evidence of sufficient efficiencies by the merging firm. Therefore, there is a double signal: the agency not only observes whether the evidence is produced, but also observes the way it has been produced. In Spence (1974) the signal (education) does not influence workers' productivity. In my model the effort level (one of the signals) is necessary to produce evidence (the other signal); the higher the effort level, the higher the probability of producing evidence. After observing the produced evidence and effort level, the agency approves the proposed merger with some probability. It is assumed that an agency's objective is to minimize mistakes in its decisions to approve or reject mergers, i.e. minimize type II and type I errors (approval of 'bad' mergers and rejection of 'good' mergers, respectively). ${ }^{9}$ By incorporating Type I and Type II errors into the agency's pay-off function we can endogenize approval probabilities through the Bayesian update mechanism.

In many laws and regulations there is fuzziness (uncertainty) in the decision making process. Rarely are there fixed and clearly specified thresholds that guarantee a certain decision. To a certain extent, laws and regulations leave it up to bureaucrats to decide each particular case. At the stage when a regulatory agency has to apply such a fuzzy rule we consider the probability of approval between zero and one (mixed strategy). Existence of some type of fuzziness in nearly every law and regulation raises the question of whether fuzziness could be beneficial for society. The answer

\footnotetext{
${ }^{7}$ For a discussion about consumer vs. total surplus approaches in merger regulation, see Neven and Roller (2005); Farrell and Shapiro (2001); Besanko and Spulber (1993); and Lyons (2002).

${ }^{8}$ Mandel (1999) discusses the recent increase in demand for economic analysis and expert witness testimony.

${ }^{9}$ Such an objective function is often assumed to characterize behavior of regulatory agencies or bureaucrats, Roller et al. (2000). In a different context it is believed that the U.S. Food and Drug Administration leans on minimization of type II errors when deciding on the approval of new drugs. However, if the agency only pays attention to type II errors, then the optimal choice would be to reject all drugs, including all 'good' ones.
} 
to this question is closely related to the possibility of commitment to certain policies on behalf of the agency.

The model presented gives new insights on different types of commitment that are available to the antitrust agency. In the case of full commitment the agency is just an executer of lawmakers' policy while in the partial commitment case the agency commits to reject all mergers when the evidence is not produced, but acts sequentially rational in other decision nodes. Ignoring some information is also a type of commitment. It is shown that if the agency can commit to act not sequentially rational in some decision nodes, then it can lower the value of the expected mistake.

To my knowledge, there is no work done on signalling in merger regulation. Only a few studies have looked at some aspects of the problem. Lagerlof and Heidhues (2005), for instance, investigated an optimal merger control regime under asymmetric information about merger-specific efficiencies. The authors did not, however, consider the possibility of the manipulation of results or cheating on behalf of 'bad' mergers. They showed that in an equilibrium 'bad' mergers would never invest in evidence production, and it is never optimal for the agency to choose a mixed strategy. However, this paper, by taking account of these strategic effects, leads to opposite results. Both merger types could produce evidence, and hence under some conditions, the agency might prefer mixed strategies to pure strategies. Besanko and Spulber (1993) examined optimal mixing between consumer and total welfare standards on behalf of the agency. If the agency were to randomize between these two standards, then merging firms would face uncertainty in the 'rules of the game'. A paper by Potters and van Winden (1992) examined lobbying under asymmetric information between a regulatory agency and a firm. In their model, the presence of a signal just shows that a firm can afford to send this signal. In the legal literature, Sanchirico (2001) presented a model of evidence production under asymmetric information when the burden of proof lies with the party of interest. His model stresses the importance of early deterrence strategies on behalf of the court or government agency in order to rely on the presented evidence. In our paper, the developed double-signal signalling model with endogenized approval probabilities allows us to analyze a more strategic interaction between the agency and merging firms.

The structure of the paper is as follows. First I describe the basic model. In Section 3 , different types of commitment that are available to the agency are analyzed. Then I proceed with a discussion of the obtained results before concluding the article. 


\section{Model}

Every merger has actual efficiencies which are not observable by the agency. However, given a market and a firm's characteristics, the agency calculates and asks to show required efficiencies that would satisfy a certain welfare standard. Thus, every merger is characterized by the difference between actual and required cost efficiencies, difference $\in(-\infty,+\infty)$. Let's consider the case of two merger types $i=\{$ Good, Bad $\}$. They have identical characteristics except one - efficiencies due to the merger. A 'good' merger has efficiencies higher than the required ones, while a 'bad' merger has efficiencies lower than required. For the simplification of further calculations I assume that 'good' and 'bad' mergers are equally distanced from the required efficiencies but only in opposite directions. For any given merger, higher efficiencies bring higher profits, $\Pi_{\text {good }}>\Pi_{\text {bad }}$. ${ }^{10}$

Both merger types want to get approval and, therefore, are ready to invest some resources to get it. Effort level $(e)$ and merger type $(i)$ determine the probability to produce evidence, $\beta_{i e}^{Y e s}$. The evidence is not produced with probability $\beta_{i e}^{\text {No }}=$ $\left[1-\beta_{i e}^{Y e s}\right]$. Assume that merging firms can choose from Zero (0), Low (L), or High (H) effort levels to produce verifiable evidence that efficiencies are sufficient. If a merging firm chooses Zero effort level, then no evidence can be produced: $\beta_{i 0}^{Y e s}=0$. Higher effort leads to higher probability to produce the evidence: $\frac{\partial \beta_{i e}^{Y e s}}{\partial e}>0, \frac{\partial^{2} \beta_{i e s}^{Y e s}}{\partial e^{2}}<0$ (as $e \rightarrow \infty, \beta \rightarrow 1)$. The same effort level gives 'good' mergers higher probability to produce the evidence: $\beta_{g e}^{Y e s}>\beta_{b e}^{Y e s}$ for $\forall e \neq 0$. One of the assumptions is that an increase in probability to produce evidence due to a higher effort level is greater for 'good' mergers than for 'bad' mergers, $\left(\beta_{g H}^{Y e s}-\beta_{g L}^{Y e s}\right)>\left(\beta_{b H}^{Y e s}-\beta_{b L}^{Y e s}\right) .{ }^{11}$ Costs of evidence production are determined by the effort level, $C(e)$, and are independent of merger type. Effort is increasingly costly: $C^{\prime}(e)>0, C^{\prime \prime}(e) \geq 0, C(0)=0$. It is assumed that effort level is perfectly correlated with success probability: the most expensive lawyer produces the best evidence.

To define equilibrium the following notation is used:

$\bar{p}(e, s)=\left(p_{0}, p_{H Y}, p_{H N}, p_{L N}, p_{L Y}\right)$ - agency's strategy - this is the vector of the agency's approval probabilities after observing effort level $e \in E:\{H, L, 0\}$ and

\footnotetext{
${ }^{10}$ This is a condition for signalling in the 'right direction', i.e. "good" firms have a larger stake in persuading a regulator than do 'bad' firms (Potters and van Winden, 1992). This condition holds in great generality: If a 'good' merger is characterized by lower costs, optimal behavior cannot yield it a lower profit.

${ }^{11}$ This assumption eliminates cases when the probability $\beta_{b e}^{Y e s}$ is close to one. Since $\beta$ is bounded, it cannot increase at a higher rate for a 'good' than for a 'bad' merger throughout ( $\beta$ converges to 1 also for a 'bad' merger).
} 
produced evidence $s \in S:\{Y$ es (evidence), No (evidence) $\}$. We do not distinguish between $p_{0 Y}$ and $p_{0 N}$ cases because 0 -effort never yields success, so $p_{0}=p_{0 Y}=p_{0 N}$. $\sigma_{i}(e)=\left(\sigma_{i}^{H}, \sigma_{i}^{L}, \sigma_{i}^{0}\right)$ - firm's strategy - this is the probability that a firm $i$ chooses effort level $e$, so that $\sum_{e} \sigma_{i}^{e}=1$.

$\beta_{i e}^{s}$ - probability of appearing at the decision node $(e, s)$ by type $i$ merger after putting in effort level $e$, where $0 \leq \beta_{i e}^{s}<1$.

$\alpha$ - agency's belief about the initial proportion of 'good' mergers and, consequently, $(1-\alpha)$ of 'bad' mergers.

A 'good' merging firm chooses an effort level by comparing expected profit:

$$
\begin{aligned}
& \mathrm{E} \Pi_{g H}=\beta_{g H}^{Y e s} p_{H Y} \Pi_{g}+\left(1-\beta_{g H}^{Y e s}\right) p_{H N} \Pi_{g}-C(H) \\
& \mathrm{E}_{g L}=\beta_{g L}^{Y e s} p_{L Y} \Pi_{g}+\left(1-\beta_{g L}^{Y e s}\right) p_{L N} \Pi_{g}-C(L) \\
& \mathrm{E}_{g 0}=p_{0} \Pi_{g}-C(0)
\end{aligned}
$$

The same comparison holds for a 'bad' merger: $\mathrm{E} \Pi_{b H}, \mathrm{E} \Pi_{b L}$, and $\mathrm{E} \Pi_{b 0}$. Therefore, the firm's maximization problem is:

$\max _{\sigma_{i}} E \Pi_{i}=\sigma_{i}^{H} E \Pi_{i H}+\sigma_{i}^{L} E \Pi_{i L}+\sigma_{i}^{0} E \Pi_{i 0}$, where $\sum_{e} \sigma_{i}^{e}=1$.

Borrowing notations from Farrell and Shapiro (1990), changes in social welfare caused by 'good' and 'bad' mergers are: $\Delta W_{\text {good }}>0$ and $\Delta W_{\text {bad }}<0$. We can assign equal weights to welfare changes due to 'bad' and 'good' mergers, $\Delta W_{g}=\Delta W_{b}$, and normalize it to 1 for the simplification of further calculations. ${ }^{12}$ The agency minimizes the value of its expected total mistake (ETM) across all decision nodes rather than a mistake in a separate decision node. ${ }^{13}$

$$
\begin{aligned}
& \min _{\bar{p}} \quad E T M=(\text { expected welfare loss due to Type I and Type II errors })= \\
& =\Delta W_{\text {good }} * \text { Prob }\left(\text { reject ' } \text { good }^{\prime} \text { merger }\right)+\Delta W_{\text {bad }} * \text { Prob }(\text { approve 'bad' merger })= \\
& =\text { Prob }(\text { reject 'good' merger })+\operatorname{Prob}\left(\text { approve } \text { bad }^{\prime} \text { merger }\right)
\end{aligned}
$$

The timing of the game is the following. Given an agency's belief about the initial proportion of 'good' and 'bad' mergers, $\alpha$ and $(1-\alpha)$, respectively, the agency draws up a rule by assigning approval probabilities conditional on the produced evidence and effort level with the goal of minimizing the expected total mistake. Knowing the rule, a merging firm chooses an effort level to maximize its expected profit. Finally, after observing a double signal, the produced evidence and effort level, the agency approves the proposed merger with the probability according to the rule. By

\footnotetext{
${ }^{12}$ Changing welfare values would change threshold values but would not change conclusions later on in the paper.

${ }^{13}$ This form of a loss function implies that the agency does not consider firms' costs of evidence production in its decisions (for example, this is the case when the agency applies a consumer surplus standard).
} 
incorporating Type I and Type II errors into the agency's pay-off function we can endogenize approval probabilities through the Bayesian update mechanism and solve the game. See Diagram 1 in Appendix B for the extensive form of the game.

\subsection{Perfect substitutability of mistakes}

In the paper I consider a case when Type I and Type II mistakes are 'perfect substitutes' ${ }^{14}$ In other words, disutilities from both types of mistakes are equal and constant. Ultimately the agency would like to enforce certain effort levels to minimize the expected total mistake, i.e. the summation of Type I and II errors across all possible decision nodes:

$$
\begin{aligned}
& \min _{\bar{p}} E T M=(\text { expected type I }+ \text { expected type II errors })= \\
& \quad=\sum_{e} \sum_{s}\left[\sigma_{g}^{e} \alpha \beta_{g e}^{s}\left(1-p_{e s}\right)+\sigma_{b}^{e}(1-\alpha) \beta_{b e}^{s} p_{e s}\right]
\end{aligned}
$$

Thus the agency cares about the distribution of 'good' and 'bad' mergers across all nodes rather than in each node separately. Given this utility function of the agency, we can define an equilibrium in the model.

Equilibrium is defined by the pair of strategies $(\sigma, p)$ which satisfy the following conditions:

1) $\sigma_{i}(e)$, where $e \in E, i=\{G o o d, B a d\}, s=\{Y e s, N o\}$ such that a firm $i$ maximizes expected profit: $\max _{\sigma_{i}} E \Pi_{i}=\sum_{e}\left[\sum_{s} \sigma_{i}^{e} \beta_{i e}^{s} p_{e s} \Pi_{i}\right]-C(e)$

2) $\bar{p}=\left(p_{0}, p_{H Y}, p_{H N}, p_{L N}, p_{L Y}\right)$ minimizes the agency's expected total mistake:

$$
\min _{\bar{p}} E T M=\sum_{e} \sum_{s}\left[\sigma_{g}^{e} \alpha \beta_{g e}^{s}\left(1-p_{e s}\right)+\sigma_{b}^{e}(1-\alpha) \beta_{b e}^{s} p_{e s}\right]
$$

3 ) the agency acts sequentially rational in each information set and updates its beliefs using the Bayesian rule.

If the agency acts sequentially rational in every decision node, then the agency makes decisions based on its beliefs about the proportion of 'good' and 'bad' mergers it faces. Let's look at a minimization problem at any decision node $(e, s)$ :

$$
\begin{aligned}
\min _{p} & {\left[\sigma_{g}^{e} \alpha \beta_{g e}^{s}\left(1-p_{e s}\right)+\sigma_{b}^{e}(1-\alpha) \beta_{b e}^{s} p_{e s}\right]=} \\
= & \sigma_{g}^{e} \alpha \beta_{g e}^{s}-p_{e s}\left[\sigma_{g}^{e} \alpha \beta_{g e}^{s}-\sigma_{b}^{e}(1-\alpha) \beta_{b e}^{s}\right]
\end{aligned}
$$

This function reaches minimum when $p_{e s}$ is equal either to 0 or 1 .

If $\left(\sigma_{g}^{e} \alpha \beta_{g e}^{s}>\left(\sigma_{b}^{e}(1-\alpha) \beta_{b e}^{s}\right)\right.$, then $p_{e s}=1$;

if the sign is " $<"$, then $p_{e s}=0$;

if the sign is $"="$, then the agency is indifferent and $p_{e s} \in[0 ; 1]$.

\footnotetext{
${ }^{14} \mathrm{~A}$ case when Type I and II errors are non-perfect substitutes is analyzed in Medvedev (2004b).
} 
Hence, if the proportion of 'good' mergers in the decision node $(e, s)$ is greater than the proportion of 'bad' mergers, then the agency is better off approving all mergers in this decision node.

\subsection{Equilibria under pure strategies only}

First, we look at the case of pure strategies on behalf of the agency, i.e. the agency can only assign the probability of 0 or 1 . In order to eliminate some trivial cases when effort levels are not affordable by one or both merger types, we introduce one assumption.

Assumption 1: If approved with probability one when the evidence is produced, then both merger types can afford any effort level, i.e. $\beta_{i e}^{Y e s} * \Pi_{i} \geq C(e)$ for $\forall e \neq 0$.

The agency's strategy is represented by the vector of approval probabilities conditional on observed signals (effort level and evidence), i.e. $\bar{p}=" p_{0} p_{H Y} p_{H N} p_{L N} p_{L Y} "$. If an agency's strategy is $\bar{p}=" 1 p_{H Y} p_{H N} p_{L N} p_{L Y}$, to approve all mergers irrespective of whether the evidence was presented or not, merging firms would always choose Zero effort level to minimize the costs because they would be approved anyway. Hence, $(1-\alpha)$ 'bad' mergers would be approved.

If the agency's strategy is " $0 p_{H Y} p_{H N} p_{L N} p_{L Y}$ ", then the possible strategies are:

\begin{tabular}{|c|c|c|c|c|}
\hline 1. "00000" & 5. " "00100" & 9. " $01000 "$ & 13. & "01100" \\
\hline$" 00001 "$ & 6. "00101" & "01001" & 14 . & "01101" \\
\hline "00010" & 7. "00110" & "01010" & 15. & "01110" \\
\hline$" 00011 "$ & 8. "00111" & "01011" & 16 . & "01111" \\
\hline
\end{tabular}

Lemma 1: Strategies 4, 8, 12, 13, and 16 imply that all mergers will be approved because given the strategy of the agency, both types would choose the same effort level (either High or Low), and the agency approves all mergers with that effort level. Proof: Given Assumption 1 and the fact that the agency disregards the firms' costs of evidence production, these strategies are equivalent to the " $1 p_{H Y} p_{H N} p_{L N} p_{L Y}$ " strategy, in which the agency ex ante decides to approve any merger.

However, there is a special case (strategy 14="01101") when High effort level is so costly that even knowing the agency would undoubtedly approve a merger after observing High effort, a merging firm would prefer Low effort level though it would be approved only if the evidence is found. Then strategy 14 could be optimal, and we should not disregard it in the further analysis. 
Lemma 2: Strategies 3, 5, 6, 7, 8, 11, and 15 imply the approval of a merger when there is no evidence and rejection of a merger when there is evidence, which makes no sense.

Proof: If such policies are implemented, then even if the evidence is produced it would be hidden. This is made clearer if we introduce one more step into the decision of merging firms: after producing evidence the firm decides whether to present it or not.

Therefore, only "00000","00001", "01000", "01001", "01101", and "11111" strategies are relevant in the analysis.

In the signalling literature there is the idea of a single-crossing property, i.e. an increase in the marginal probability to produce the evidence is higher for 'good' mergers than for 'bad' mergers: $\frac{\partial \beta_{g e}^{Y e s}}{\partial e}>\frac{\partial \beta_{b e}^{Y e s}}{\partial e}\left(\right.$ or $\left.\frac{\beta_{g H}^{Y e s}}{\beta_{g L}^{Y e s}}>\frac{\beta_{b H}^{Y e s}}{\beta_{b L}^{Y e s}}\right)$. In the paper, we distinguish cases with and without this property.

For simplicity we introduce the following notation:

$\beta_{g H}^{Y e s}=a \quad \beta_{g L}^{Y e s}=c \quad \beta_{b H}^{Y e s}=b \quad \beta_{b L}^{Y e s}=d$

In order to derive optimal policies in the model, two lemmas are needed:

Lemma 3: The single-crossing property $\frac{a}{c}>\frac{b}{d}$ guarantees that $(a-c)>(b-d)$. Proof: If $\frac{a}{c}>\frac{b}{d} \Rightarrow\left(\frac{a}{c}-1\right)>\left(\frac{b}{d}-1\right) \Rightarrow \frac{a-c}{c}>\frac{b-d}{d}$, because $c>d$, then $a-c>b-d$.

Lemma 4: If $\frac{a}{c}>\frac{b}{d}$, then strategy "00001" is never optimal for the agency. Proof: See Appendix A.

The latter lemma states that if $\frac{a}{c}>\frac{b}{d}$ and given Assumption 1, strategy "00001" is always dominated either by strategy "01000" or by strategy "00000". Now we can derive equilibrium strategies given an initial belief about $\alpha$, proportion of 'good' mergers $^{15}$ :

Equilibrium 1 (E1): If $\frac{a}{c}>\frac{b}{d}$, then equilibrium is:

a) $\alpha \in\left[0, \alpha_{1}\right)$, then $\bar{p}=(0,0,0,0,0), \sigma_{g}=(0,0,1), \sigma_{b}=(0,0,1), E T M=\alpha$

b) $\alpha \in\left[\alpha_{1}, \alpha_{2}\right)$, then $\bar{p}=(0,1,0,0,0), \sigma_{g}=(1,0,0), \sigma_{b}=(1,0,0)$, $E T M=\alpha(1-a)+(1-\alpha) b$

c) $\alpha \in\left[\alpha_{2}, 1\right]$, then $\bar{p}=(1,1,1,1,1), \sigma_{g}=(0,0,1), \sigma_{b}=(0,0,1), \operatorname{ETM}=(1-\alpha)$ where $\alpha_{1}=\frac{b}{a+b}$ and $\alpha_{2}=\frac{1-b}{2-a-b}$

\footnotetext{
${ }^{15}$ I will disregard "knife-edge" cases, such as $a \Pi_{g}-C(H)=c \Pi_{g}-C(L), b \Pi_{b}-C(H)=d \Pi_{b}-C(L)$, $\frac{a}{c}=\frac{b}{d}$ because these equalities come entirely from values of exogenous parameters rather than from rational choice of probabilities by the agency to enforce mixing on behalf of firms. The probability of such events (equalities) is zero.
} 
Every strategy can be represented by a line (see Graph 1 in Appendix C). For example, if the agency's strategy is "01000", i.e. to approve a merger only after observing the produced evidence and high effort level, then both merger types choose High effort, and some of them will succeed in producing the evidence. The expected total mistake is equal to $\alpha(1-a)+(1-\alpha) b$, where $\alpha(1-a)$ is Type I error, i.e. rejected 'good' mergers that were not able to produce the evidence, and $(1-\alpha) b$ is Type II error, i.e. approved 'bad' mergers that were able to produce the evidence. In Graph 1 , the lowest line (strategy) reflects the optimal strategy for given values of $\alpha$. When $\alpha$ is small, the agency believes that there are too few 'good' mergers and rejects all mergers. Both merger types choose zero effort level to minimize costs. With intermediate values of $\alpha$, the agency approves mergers only if evidence is produced after High effort level but rejects them otherwise. This happens because according to Proposition 1 the agency prefers to enforce High effort level to produce the evidence. Both merger types choose High effort level, which is affordable according to Assumption 1. When $\alpha$ is big, then the agency approves all mergers irrespective of whether the evidence is produced or not, and both merger types choose Zero effort level to minimize costs.

Equilibrium 2 (E2): If $\frac{a}{c}<\frac{b}{d}$, then equilibrium is:

a) $\alpha \in\left[0, \alpha_{1}\right)$, then $\bar{p}=(0,0,0,0,0), \sigma_{g}=(0,0,1), \sigma_{b}=(0,0,1), E T M=\alpha$

b) $\alpha \in\left[\alpha_{1}, \alpha_{2}\right)$, then $\bar{p}=(0,0,0,0,1), \sigma_{g}=(0,1,0), \sigma_{b}=(0,1,0)$, ETM $=\alpha(1-c)+(1-\alpha) d$

c) $\alpha \in\left[\alpha_{2}, \alpha_{3}\right)$, then $\bar{p}=(0,1,0,0,0), \sigma_{g}=(1,0,0), \sigma_{b}=(1,0,0)$, $E T M=\alpha(1-a)+(1-\alpha) b$

d) $\alpha \in\left[\alpha_{3}, 1\right]$, then $\bar{p}=(1,1,1,1,1), \sigma_{g}=(0,0,1), \sigma_{b}=(0,0,1), \operatorname{ETM}=(1-\alpha)$ where $\alpha_{1}=\frac{d}{c+d}, \quad \alpha_{2}=\frac{b-d}{a-c+b-d}, \quad \alpha_{3}=\frac{1-b}{2-a-b}$

If $\frac{a}{c}<\frac{b}{d}$, then for certain values of $\alpha$ there is one more optimal strategy "00001" (the agency approves mergers only if evidence is produced after Low effort level but rejects them otherwise). Both merger types choose Low effort level, which is affordable according to Assumption 1. This additional equilibrium strategy arises because although the shift from Low to High effort level increases the probability to produce the evidence for 'good' mergers more than for 'bad' ones, $(a-c)>(b-d)$, but not as much as when $\frac{a}{c}>\frac{b}{d}$ (see Graph 2 in Appendix C).

In all cases above, the agency acts sequentially rational in every decision node, i.e. the agency makes decisions based on its beliefs about the proportion of 'good' and 'bad' mergers it faces. 


\subsection{Equilibria if mixing is possible}

Now we look at whether by applying a fuzzy approval rule (mixed strategies) the agency can do as well or better as under pure strategies. As we saw in Section 2.1, mixing is possible when there are equal proportions of 'good' and 'bad' merger types in a decision node, i.e. when the agency is indifferent between approving or rejecting a merger. Such a result comes from the assumptions about the perfect substitutability of different types of mistakes and equal weights to welfare changes due to 'good' and 'bad' mergers. Then the proposition follows that it is impossible to have equal proportions of 'good' and 'bad' mergers in any two nodes simultaneously with the exception of one case.

\section{Lemma 5:}

Given assumptions $a>b>d$ and $a>c>d$, mixing in any two decision nodes simultaneously is impossible besides the strategy $\bar{p}=\left(0,1, p_{H N}, 0,0, p_{L Y}\right)$.

Now using Proposition 2 and the definition of the equilibrium from Section 2.1, we can derive the following mixing equilibria (ME) (for all values see Appendix A):

ME1: $\bar{p}=\left(0, p_{H Y}, 0,0,0\right), \sigma_{g}=(1,0,0), \sigma_{b}=\left(\sigma_{b}^{H}, 0,\left[1-\sigma_{b}^{H}\right]\right), E T M=\alpha$

ME2: $\bar{p}=\left(0,0,0,0, p_{L Y}\right), \sigma_{g}=(0,1,0), \sigma_{b}=\left(0, \sigma_{b}^{L},\left[1-\sigma_{b}^{L}\right]\right), E T M=\alpha$

ME3: $\bar{p}=\left(0,1,1, p_{L N}, 1\right), \sigma_{g}=\left(\sigma_{g}^{H},\left[1-\sigma_{g}^{H}\right], 0\right), \sigma_{b}=(0,1,0), \operatorname{ETM}=1-\alpha$

ME4: $\bar{p}=\left(0,1, p_{H N}, 0, p_{L Y}\right), \sigma_{g}=\left(\sigma_{g}^{H},\left[1-\sigma_{g}^{H}\right], 0\right), \sigma_{b}=\left(\sigma_{b}^{H},\left[1-\sigma_{b}^{H}\right], 0\right)$, $E T M=\alpha(1-a)+(1-\alpha) b$

ME5: $\bar{p}=\left(0,1,1,0, p_{L Y}\right), \sigma_{g}=\left(\sigma_{g}^{H},\left[1-\sigma_{g}^{H}\right], 0\right), \sigma_{b}=(0,1,0), \operatorname{ETM}=(1-\alpha) \frac{d}{c}$.

As an example, we look at ME1. When $\alpha$ is small, the agency can assign nonzero probability to the (High efforts, Yes evidence) decision node. The assigned probability makes 'bad' mergers indifferent between High and Zero effort levels and 'bad' mergers can mix, while 'good' mergers choose High efforts to produce the evidence with probability one. ${ }^{16}$ Bad mergers mix with a probability that makes an equal proportion of 'good' and 'bad' mergers (50/50) in the decision node $\left(e^{H}, Y e s\right)$. If 'good' and 'bad' mergers are in equal proportions, then the agency is indifferent between approving or rejecting them and can choose any probability between 0 and 1. In the equilibrium it chooses $p_{H Y}$ such that makes a 'bad' merger indifferent. The value of the expected total mistake is $(\alpha)$, i.e. the same as under strategy $(00000)$, because the agency decreases the number of rejected 'good' mergers (Type I error) from $\alpha$ to $\alpha-\alpha a p_{H Y}$ but increases the number of approved 'bad' mergers (Type II

\footnotetext{
${ }^{16} \mathrm{~A}$ 'good' merger reaches first a "point of profitability", i.e. condition when an effort level becomes profitable for a merging firm. It follows from assumptions $\Pi_{g}>\Pi_{b}$ and $a>b$ and $c>d$.
} 
error) from 0 to $(1-\alpha) \sigma_{b}^{H} b p_{H Y}$. The same type of trade-off between Type I and Type II errors can be shown for other Mixing Equilibria ME2-ME4, i.e. the agency is not better off applying a fuzzy approval rule.

However, Equilibrium ME5 is of prime interest to us. If $\Pi_{\text {good }}$ is relatively large compared to $\Pi_{b a d}$ as well as the difference between High and Low effort costs and the difference in success probabilities to produce the evidence, $d$ and $c$, then the agency can assign such probability $p_{L Y}^{M E 4}$ that 'good' mergers would mix between High and Low effort levels while all 'bad' ones would choose Low efforts. In other words the equilibrium exists if the following conditions on exogenous parameters are satisfied: $p_{L Y}^{M E 5}$ is such that $\Pi_{g}-C(H)=p_{L Y}^{M E 5} c \Pi_{g}-C(L)$ and $\Pi_{b}-C(H)<p_{L Y}^{M E 5} k \Pi_{b}-C(L)$.

Proposition 1: Mixing Equilibrium ME5 gives a lower value of expected total mistake than:

a) E1 on the interval $\left(\frac{d-c b}{d-c b+c(1-a)} ; 1\right)$ if $\frac{a}{c}>\frac{b}{d}$;

b) E2 on the interval $\left(\frac{d}{c+d} ; 1\right)$ if $\frac{a}{c}<\frac{b}{d}$.

This is the only mixing equilibrium when a fuzzy approval rule decreases the expected total mistake, while the agency acts sequentially rational in every decision node (see Graph 3 in Appendix C).

\section{Different types of commitment}

The ultimate goal of the agency is to distinguish between 'good' and 'bad' merger types by choosing a vector of probabilities to minimize the value of the total expected mistake. One way to distinguish merger types is to choose such probabilities that 'good' mergers would extract the maximum effort level to produce evidence, while 'bad' mergers would find it either unprofitable to produce the evidence or would choose a low effort level. In the model, this is equivalent to the situation where 'good' mergers should choose High effort level while 'bad' mergers should choose Zero or Low effort level. However, assigning an pre-determined vector of probabilities, which minimizes the agency's mistakes, could lead to the situation when the agency wants to deviate from its ex-ante strategy. This raises a commitment problem. 


\subsection{Pure strategies and no attention to effort level}

In this section, we compare the situation in which the agency does not pay attention to the way the evidence is produced with the situation from the previous section in which there is attention to the effort level. The way to make the comparison is to assume that the agency knows all the probabilities for both merger types under Low and High effort levels; however, it does not use this information in distinguishing merger types. Its decisions are based only on whether the evidence is produced or not. In this case, the equilibrium is defined in the following way:

Equilibrium is defined by the pair of strategies $(\sigma, p)$, which satisfy the following conditions:

1) $\sigma_{i}(e)$, where $e \in E, i=\{G o o d, B a d\}, s=\{Y e s, N o\}$ such that a firm $i$ maximizes expected profit: $\max _{\sigma_{i}} E \Pi_{i}=\sum_{e}\left[\sum_{s} \sigma_{i}^{e} \beta_{i e}^{s} p_{s} \Pi_{i}\right]-C(e)$

2) $\bar{p}=\left(p_{0}, p_{\text {yes }}, p_{n o}\right)$ minimizes the agency's expected total mistake:

$\min _{\bar{p}} \quad E T M=\sum_{e} \sum_{s}\left[\sigma_{g}^{e} \alpha \beta_{g e}^{s}\left(1-p_{s}\right)+\sigma_{b}^{e}(1-\alpha) \beta_{b e}^{s} p_{s}\right]$

3 ) the agency acts sequentially rational at each information set and updates its beliefs using the Bayesian rule while consciously ignoring the information about the effort level.

Given this definition of the equilibrium the agency can choose the following strategies. It can approve or reject all mergers, and consequently, both merger types would choose Zero effort level to minimize costs. For intermediate values of $\alpha$, the agency can approve mergers if evidence is produced and reject them otherwise. In the latter case, the profitability of different effort levels for merging firms should be considered, i.e. what effort level is more profitable for them given a merger is approved with the probability of one when the evidence is produced. There are three possible cases: both merger types prefer High or Low effort levels or the case in which High effort level is more profitable for a 'good' merger, while Low effort level is more profitable for a 'bad' merger. ${ }^{17}$

Considering these three cases, we can derive equilibrium strategies given initial beliefs about the proportion of 'good' mergers, $\alpha$. In the cases when both merger types prefer High or Low effort levels, the inability to enforce a certain effort level will lead to a higher expected mistake than in equilibria E1 and E2 for some intermediate values of $\alpha$ and the same mistake for all other values of $\alpha$. For a visual representation see Graph 1 and 2 in Appendix C. ${ }^{18}$

\footnotetext{
${ }^{17}$ It is not possible that a 'bad' merger prefers High effort level while a 'good' merger prefers Low level because $\Pi_{g}>\Pi_{b}$ and $(a-c)>(b-d)$.

${ }^{18}$ See Medvedev (2004b) for all formal derivations.
} 
However, when High effort level is more profitable for a 'good' merger, while Low effort level is more profitable for a 'bad' merger, given a merger is approved with the probability of one when the evidence is produced, i.e. $a \Pi_{g}-C(H)>c \Pi_{g}-C(L)$ and $b \Pi_{b}-C(H)<d \Pi_{b}-C(L)$, then Equilibrium 3 (E3) is:

a) $\alpha \in\left[0, \alpha_{1}\right)$, then $\bar{p}=(0,0,0), \sigma_{g}=(0,0,1), \sigma_{b}=(0,0,1), \operatorname{ETM}=\alpha$

b) $\alpha \in\left[\alpha_{1}, \alpha_{2}\right)$, then $\bar{p}=(0,1,0), \sigma_{g}=(1,0,0), \sigma_{b}=(0,1,0)$, ETM $=\alpha(1-a)+(1-\alpha) d$

c) $\alpha \in\left[\alpha_{2}, 1\right]$, then $\bar{p}=(1,1,1), \sigma_{g}=(0,0,1), \sigma_{b}=(0,0,1), \operatorname{ETM}=(1-\alpha)$ where $\alpha_{1}=\frac{d}{a+d}$ and $\alpha_{2}=\frac{1-d}{2-a-d}$.

Given these equilibrium strategies, we can compare this outcome with the outcome where the agency pays attention to effort levels:

\section{Proposition 2:}

a) E3 (equilibrium with no attention to effort level) gives a smaller expected total mistake than either E1 or E2 on the interval $\alpha \in\left[\frac{d}{a+d}, \frac{1-d}{2-a-d}\right)$ and the same mistake for other values of $\alpha$.

For a visual representation see Graph 4 in Appendix C. Equilibrium strategy E3b could be called a "voluntary separation": High effort level is more profitable for a 'good' merger, while Low effort level is more profitable for a 'bad' merger. For the intermediate values of $\alpha$, the agency knows that only 'bad' mergers would prefer Low effort level and that some of them would succeed in producing evidence. If effort levels are considered in the decision-making, the agency would assign probability zero to the decision node $\left(e^{L}, Y e s\right)$, i.e. $p_{L Y}=0$. However, knowing this, a 'bad' merger would choose High effort level so as not to be distinguished by the agency, and according to Assumption 1, it can afford this effort level. The shift in the effort level by a 'bad' merger would lead to the situation in which 'bad' mergers would invest more resources into evidence production. Consequently, more 'bad' mergers would have produced the evidence and, as a result, a bigger mistake by the agency.

Thus we can say that a rule to approve all mergers which produced evidence is optimal under some parameters, yet enforcing certain effort levels could lower the expected total mistake. However, under some conditions, even recognizing potentially harmful mergers, the agency is better off approving rather than blocking them; otherwise, a 'bad' merger would mimic the choice of a 'good' merger. The case of voluntary separation shows that sometimes the use of extra information (the way the evidence is produced) leads to an undesirable outcome (higher expected total mistake). This contradicts the usual perception that the more information used the better. 


\subsection{Full and partial commitments}

One type of commitment we discussed in the section above is where the agency commits not to pay attention to the way the evidence was produced. Another type of commitment is to assume that lawmakers write down the agency's rule, which minimizes the expected total mistake, and then the agency automatically applies this rule $^{19}$. This is termed full commitment. In the case of full commitment the agency is just an executor of lawmakers' policy. Lawmakers draw up a policy and the agency automatically applies it no matter what its beliefs. The lawmakers acting as a social planner assign probabilities to each decision node with the goal of minimizing the expected total mistake: 'good' mergers extract a maximum effort level to produce the evidence, while 'bad' mergers find it either unprofitable to produce the evidence at all or choose Low effort level. There are probabilities that make a 'bad' merger indifferent between two effort levels, the assigned probabilities should be by a fraction smaller than these (by $\varepsilon$, where $\varepsilon \rightarrow 0$ ) to guarantee that 'bad' mergers stay at Low or Zero effort levels while the share of rejected 'good' mergers is minimized. Let's consider one possible case to estimate the approximate benefits for the agency that can come from full commitment. If the agency can fully commit to certain probabilities then the full commitment equilibrium (FCE) strategies for the case $\frac{C(H)}{C(L)}>\frac{b c}{a d}$ are the following ${ }^{20}$ :

a) $\alpha \in\left[0, \alpha_{1}\right), \bar{p}=\left(0, p_{H Y}^{F C}, 0,0,0\right), \sigma_{g}=(1,0,0), \sigma_{b}=(0,0,1), E T M=\alpha-\alpha a p_{H Y}^{F C}$

b) $\alpha \in\left[\alpha_{1}, \alpha_{2}\right]$, then $\bar{p}=\left(0,1,0,0, p_{L Y}^{F C}\right), \sigma_{g}=(1,0,0), \sigma_{b}=(0,1,0)$,

$E T M=\alpha(1-a)+(1-\alpha) d p_{L Y}^{F C}$

c) $\alpha \in\left(\alpha_{2}, 1\right]$, then $\bar{p}=\left(0,1,1, p_{L N}^{F C}, 1\right), \sigma_{g}=(1,0,0), \sigma_{b}=(0,1,0)$,

$E T M=(1-\alpha) d+(1-\alpha)(1-d) p_{L N}^{F C}$

See Appendix A for $\alpha_{1}, \alpha_{2}, p_{H Y}^{F C}, p_{L N}^{F C}, p_{L Y}^{F C}$. Probabilities are such that 'good' mergers choose High effort level, while 'bad' mergers choose Zero or Low effort level. This separation is the best the agency can do under such parametrization. Such a policy brings the lowest total mistake for $\alpha \in(0,1)$ (see Graph 5 in Appendix C). However, it is not a sequentially rational equilibrium. The agency would like to deviate expost from the assigned probabilities, because in certain decision nodes there will be only 'good' or 'bad' mergers and optimal approval probabilities will be one or zero,

\footnotetext{
${ }^{19}$ Usually the commitment problem is resolved by delegating decisions to some independent body with a different utility function. See for example Cukierman (1994) central bank and inflation rate and Melumad and Mookherjee (1989) government and tax collection.

${ }^{20}$ If $\frac{C(H)}{C(L)}<\frac{b c}{a d}$ the initial strategy (strategy FCEa) would be $\bar{p}=\left(0,0,0,0, \tilde{p}_{L Y}\right), \sigma_{g}=$ $(0,1,0), \sigma_{b}=(0,0,1)$, where $\tilde{p}_{L Y}$ makes it profitable only for a 'good' merger to choose $e^{L}$ (see Appendix A).
} 
respectively.

It is interesting to compare the case of full commitment with that of partial commitment, where the agency has some freedom and sometimes makes a decision based on its beliefs after observing effort level and evidence. One partial commitment could be such that there are precise instructions for the agency to follow to reject all mergers when the evidence is not produced, while the agency has freedom to apply a probability between zero and one when the evidence is produced, i.e. to act sequentially rational in some decision nodes. If a commitment $p(e, N o)=p_{H N}=p_{L N}=0$, then under some parameters there is a partial commitment equilibrium (PCE) strategy:

$\bar{p}=\left(0,1,0,0, p_{L Y}^{P C}\right), \sigma_{g}=\left(\sigma_{g}^{H},\left[1-\sigma_{g}^{H}\right], 0\right), \sigma_{b}=(0,1,0)$,

$E T M=\alpha \sigma_{g}^{H}(1-a)+\alpha\left(1-\sigma_{g}^{H}\right)\left(1-c p_{L Y}^{P C}\right)+(1-\alpha) d p_{L Y}^{P C}$

where $\sigma_{g}^{H}=\left[1-\frac{(1-\alpha)}{\alpha} \frac{d}{c}\right] \in[0 ; 1]$, i.e. $\alpha \in\left[\frac{d}{c+d} ; 1\right]$,

$$
p_{L Y}^{P C}=\frac{1}{c}\left(a-\frac{C(H)-C(L)}{\Pi_{g}}\right) \text { and } p_{L Y}^{P C}: 0<b \Pi_{b}-C(H)<p_{L Y}^{P C} d \Pi_{b}-C(L)
$$

The agency can choose probability $p_{L Y}$, which makes a 'good' merging firm indifferent between High and Low effort levels and opens the possibility for mixing. A 'good' firm can mix in the proportion that makes the agency indifferent between approving or rejecting mergers in the decision node $\left(e^{L}, Y e s\right)$, i.e. when there are equal proportions of 'good' and 'bad' mergers in this decision node. As long as the agency is indifferent, it can choose probability $p_{L Y}$ that makes a 'good' firm indifferent between High and Low. Approval probability $p_{L Y}$ should be such that Low effort level is more profitable for 'bad' mergers than High effort level.

The problem with such an equilibrium is that the agency knows that only 'good' mergers are in the $\left(e^{H}, N o\right)$ decision node. There is, hence, an incentive to deviate and approve all mergers in this decision node. However, if the commitment to reject all mergers that have not produced evidence is credible, then the agency might have a lower expected total mistake for some values of parameters.

\section{Proposition 3:}

If the commitment to reject all mergers that have not produced the evidence is credible and $\frac{a}{c}<\frac{b}{d}$, then equilibrium strategy $\left(0100 p_{L Y}^{P C}\right)$ gives a smaller expected total mistake than E2 on the interval $\alpha \in\left(\frac{d}{c+d} ; \frac{1-a \frac{d}{c}}{2-a-a \frac{d}{c}}\right)$.

See Graph 6 in Appendix C. From the practical point of view, it is easier to commit to and legally enforce zero or one probability as in the case of partial commitment than to any probability in between as in the case of full commitment. 


\subsection{Application fees}

As we saw in the previous cases of commitments, the separation of merger types leads to a lower expected mistake. In the full commitment equilibrium FCE(a), i.e. when $\alpha$ is relatively small, the agency chooses such mixing probability that a 'good' merger opts for High effort level, while a 'bad' merger decided not to produce the evidence and consequently does not propose to merge. However, the agency can achieve the same result without mixing by imposing an application fee on all firms that are willing to merge and to be involved in evidence production.

Proposition 4: If the agency commits to probability zero when the evidence is not produced and an application fee is $F=b \Pi_{b}-C(H)+\varepsilon$, where $\varepsilon \rightarrow 0$, then the expected total mistake will be lower than in equilibria E1(a), E2(a), PCE(a), and FCE(a).

Merger types make the decision to go into evidence production and to choose High effort level whenever the following inequalities hold: $\left[a p_{H Y}+(1-a) p_{H N}\right] \Pi_{g}-C(H)>$ 0 and $\left[b p_{H Y}+(1-b) p_{H N}\right] \Pi_{b}-C(H)>0$. If the agency commits to probability zero when the evidence is not produced $\left(p_{H N}=0\right)$, then the agency should choose such a fixed fee $(\mathrm{F})$ that makes evidence production unprofitable for a 'bad' merger, i.e. $F=b \Pi_{b}-C(H)+\varepsilon$, where $\varepsilon \rightarrow 0$. Since a 'good' merger has higher incentives, $\Pi_{g}>\Pi_{b}$, and higher chances to produce the evidence, $a>b$, then only 'good' mergers would appear in the decision node $\left(e^{H}, Y e s\right)$, and acting sequentially rational the agency will assign probability one to this node. Thus the expected total mistake will be $\alpha(1-a)$, which is always lower than in equilibria E1(a), E2(a), PCE(a), and $\mathrm{FCE}(\mathrm{a})$.

\section{Discussion and Conclusion}

The presented signalling model allows us to look at some effects of the inclusion of an efficiency defense in merger regulation. An overly intense effort to produce evidence could be a sign that 'good' mergers are trying to increase their chances to get approval and benefit financially from the merger. Hence, a high effort level could be a signal about their efficiencies. 'Bad' merger types, on the other hand, could be putting more efforts than is optimal for them to mimic 'good' mergers and to be approved. The prime difference from Spence's (1974) and Potters and van Winden's (1992) models is that the choice of the effort level effects the probability of success in producing the evidence, as opposed to a mere financial ability to send a signal. 
Overall, the antitrust agency can decrease the value of the total expected mistake in its decisions by paying attention to the effort level with which the evidence is produced. In practice, the agency could select a small number of consulting and law firms (extremely expensive) or create a governmental agency (relatively cheap but inefficient) and accept the evidence only from them.

In the paper, the agency uses mixed strategies in accepting efficiency defense arguments after observing certain signals as a tool to (partially) separate different merger types by changing approval probabilities and, consequently, firms' expected payoffs from a merger. The separation leads to a lower value of the expected mistake by the agency.

The present analysis shows that if Type I and Type II mistakes are perfect substitutes, then only under strict restrictions on exogenous parameters could fuzziness be a rational choice of a regulatory agency (ME5). However, if the agency can commit to certain probabilities (i.e. not to act sequentially rational in some decision nodes), then it can lower the value of the expected mistake. In the case of full commitment, the agency is just an executer of lawmakers' policy, while in the partial commitment case the agency commits to reject all mergers when the evidence is not produced, but acts sequentially rational in other decision nodes. In both cases the the agency is better off applying a fuzzy approval rule for some parameters. From the practical point of view it is easier to commit to and legally enforce zero or one probability as in the case of partial commitment than to any probability in between as in the case of full commitment. Introduction of application fees for merging firms could be a useful instrument to separate merger types and to lower expected mistake as long as commitment to reject all mergers when the evidence is not produced is possible. This policy is relatively easy to implement as well. Ignoring some information is a type of commitment. For some parameters the agency is better off approving harmful mergers rather than blocking them; otherwise, 'bad' mergers would invest more resources into evidence production and, as a result, a bigger mistake is made by the agency. Hence, Proposition 2 gives a value to the commitment of not using the information.

In this paper we consider the case of a perfect substitutability of different types of mistakes. However, the agency repeatedly faces mergers in each decision node and may be the agency prefers to have a balanced composition of different types of mistakes in its approval/rejection history. The idea is that the agency might bear extra costs by making too many Type I or Type II errors in a particular decision node. In the working paper (Medvedev 2004b) it was shown that the change in the 
form of agency's objective function could lead to the emergence of fuzzy approval rules under a wider range of exogenous parameters ${ }^{21}$.

There is a long-running discussion about the feasibility of mixed strategies in real life. Rubinstein (1991) provides an overview of the rationale behind mixed strategies. One of them is Harsanyi's (1973) idea of purification; in this context, mixing probabilities represent a distribution of preferences among bureaucrats within the agency. One can argue that really individual bureaucrats do not play mixed strategies, but that they slightly differ and, hence, because cases are randomly allocated to bureaucrats, the result is as if the agency plays a mixed strategy. At the same time, in some equilibria, merging firms use mixed strategies between different effort levels. One of the explanations of why a firm would mix in a fixed proportion (say, with $40 \%$ High effort level and with $60 \%$ Low effort level) could be that the 40-60 proportion reflects a divide between risk-loving and risk-averse people. Although both efforts give equal expected payoff, risk-averse agents would choose High effort level and be approved with probability 1 if the evidence is produced, while risk-lovers would choose Low effort level and be approved with probability less than one.

On the one hand, the idea that the agency is "flipping a coin" while accepting efficiency defense arguments ${ }^{22}$ is hard to sell to lawyers, who request a consistency in decisions (i.e. the agency can play only pure strategies), but, on the other hand, if such behavior enhances the agency's welfare, I do not see why it should be excluded from the analysis. The paper shows that a regulatory agency could prefer to maintain some randomization while making approval decisions.

\section{References}

[1] Besanko D., D.Spulber, 1993. "Contested mergers and equilibrium antitrust policy", Journal of Law, Economics and Organization 9(1), 1-29.

[2] Cukierman A., 1994. "Commitment through Delegation, Political Influence and Central Bank Independence", Kluwer Academic Publishers, 1994.

[3] Deneckere R., C.Davidson, 1985. "Incentives to form coalitions with Betrand competition", Rand Journal of Economics 16, 473-86.

\footnotetext{
${ }^{21}$ If different types of mistakes are non-perfect substitutes (for example, quadratic disutility from both types of mistake), then there can be a global mixing equilibrium when both firms mix High and Low effort levels and the agency is mixing at each decision node.

${ }^{22}$ Motta (2004) describes some inconsistencies in accepting efficiency defense arguments; Sanchirico (1997) discusses uncertainty in the court's final assessments.
} 
[4] Farrell J., C.Shapiro, 1990. "Horizontal mergers: an equilibrium analysis", American Economic Review 80, 107-26

[5] Farrell J., C.Shapiro, 2001. "Scale economies and synergies", Antitrust Law Journal 68(3), 685-710.

[6] Harsanyi J., 1973. "Games with randomly distributed payoffs: a new rationale for mixed strategy equilibrium points", International Journal of Game Theory $3,211-225$.

[7] Kamien M., I.Zang, 1990. "The limits of monopolization through acquisition", Quarterly Journal of Economics, Vol. CV, 456-500.

[8] Lagerlof J., P.Heidhues, 2005. "On the desirability of an efficiency defense in merger control", International Journal of Industrial Organization 23, 803-827.

[9] Lyons B., 2002. "Could politicians be more right than economists? A theory of merger standards", CCR Working Paper No. 02-1.

[10] Mandel M., 1999. "Going for the gold: economists as expert witnesses", Journal of Comparative Economics 13(2), 113-20.

[11] Medvedev A., 2004a. "Structural remedies in merger regulation in a Cournot framework", CERGE-EI Working Paper No.229.

[12] Medvedev A., 2004b. "Efficiency defense and administrative fuzziness in merger regulation", CERGE-EI Working Paper No.234.

Available at: http://www.cerge-ei.cz/pdf/wp/Wp234.pdf

[13] Melumad N., Mookherjee D., 1989. "Delegation as commitment: the case of income tax audits", Rand Journal of Economics 20, 139-169.

[14] Motta M., 2004. "Competition policy: theory and practice", Cambridge University Press, 2004.

[15] Neven D., L.Roller, 2005. "Consumer surplus vs. welfare standard in a political economy model of merger control", International Journal of Industrial Organization 23, 829-848.

[16] Perry M., R.Porter, 1985. "Oligopoly and the incentive for horizontal merger", American Economic Review 75(1), 219-27.

[17] Potters J., F. van Winden, 1992. "Lobbying and assymetric information", Public Choice 74, 269-92. 
[18] Roller L., J.Stennek, and F.Verbonen, 2000, "Efficiency gains from mergers", WZB discussion paper FS IV 00-09.

[19] Rubinstein A., 1991. "Comments on the interpretation of game theory", Econometrica 58(4), 909-924.

[20] Salant S., S.Switzer, and R.Reynolds, 1983. "Losses from horizontal merger: the effects of an exogenous change in industry structure on Cournot-Nash equilibrium", Quarterly Journal of Economics 98(2), 185-199.

[21] Sanchirico W., 1997. "The burden of proof in civil litigation: A simple model of mechanism design", Internation Review of Law and Economics 17, 431-447.

[22] Sanchirico W., 2001. "Relying on the information of interested and potentially dishonest parties", American Law and Economics Review 3(2), 320-357.

[23] Spence M., 1974. "Market signaling: information transfer in hiring and related screening process". Cambridge, MA: Harvard University Press.

[24] Williamson O., 1968. "Economics as an antitrust defence: the welfare trade-off", American Economic Review 58(1), 18-36

[25] Werden G., 1996. "A robust test for consumer welfare enhancing mergers among sellers of differentiated products", Journal of Industrial Economics 44(4), 409413.

[26] Yao D., T.Dahdouh, 1993. "Information problems in merger decision making and their impact on development of an efficiencies defense", Antitrust Law Journal 62(1), 23-45.

\section{Appendix A: Proofs and Calculations}

As a reminder: $\quad \beta_{g H}^{Y e s}=a \quad \beta_{g L}^{Y e s}=c \quad \beta_{b H}^{Y e s}=b \quad \beta_{b L}^{Y e s}=d$

\section{Lemma 4:}

If $\frac{a}{c}>\frac{b}{d}$, then strategy $2=(00001)$ is never optimal for the agency.

Proof: In other words, if $\frac{a}{c}>\frac{b}{d}$, then strategy 2 is always dominated either by strategy 9 or by strategy 1, i.e "00001" $\succ$ "01000" and "00001" $\succ$ "00000" is impossible. The payoff of the agency under strategy "00001" is $\alpha(1-c)+(1-\alpha) d$, the payoff under strategy " $01000 "$ is $\alpha(1-a)+(1-\alpha) b$, and the payoff under strategy "00000" is $\alpha$. 
$\left\{\begin{array}{l}\alpha(1-c)+(1-\alpha) d<\alpha \\ \alpha(1-c)+(1-\alpha) d<\alpha(1-a)+(1-\alpha) b\end{array} \Leftrightarrow\left\{\begin{array}{l}\alpha>\frac{d}{c+d} \\ \alpha<\frac{b-d}{a+b-c-d}\end{array}\right.\right.$
$\Leftrightarrow \frac{b-d}{a+b-c-d}>\alpha>\frac{d}{c+d} \Leftrightarrow \frac{b-d}{a+b-c-d}>\frac{d}{c+d} \Leftrightarrow \frac{a}{c}<\frac{b}{d}$. It is a contradiction.

\section{Lemma 5:}

Given assumptions $a>b>d$ and $a>c>d$, mixing in any two decision nodes simultaneously is impossible, besides the strategy $\left(01 p_{H Y} 0 p_{L Y}\right)$.

Proof: Let $\mu_{H Y}, \mu_{H N}, \mu_{L Y}, \mu_{L N}$ be proportions of 'good' mergers in respective decision nodes. Mixing is possible when there are equal proportions of 'good' and 'bad' merger types in a decision node.

a) Assume $\mu_{H Y}=\mu_{H N} \Leftrightarrow \frac{\alpha \sigma_{g} a}{\alpha \sigma_{g} a+(1-\alpha) \sigma_{b} b}=\frac{\alpha \sigma_{g}(1-a)}{\alpha \sigma_{g}(1-a)+(1-\alpha) \sigma_{b}(1-b)} \Leftrightarrow$ $\Leftrightarrow \alpha^{2} \sigma_{g} a(1-a)+\alpha(1-\alpha) \sigma_{b} a(1-b)=\alpha^{2} \sigma_{g} a(1-a)+\alpha(1-\alpha) \sigma_{b}(1-a) b \Leftrightarrow$ $a(1-b)=(1-a) b \Leftrightarrow a=b$. It is a contradiction, and consequently $\mu_{H Y} \neq \mu_{H N}$.

Analogously $\mu_{L Y} \neq \mu_{L N}$.

b) Assume $\mu_{H Y}=\mu_{L Y} \Leftrightarrow \frac{\alpha \sigma_{g} a}{\alpha \sigma_{g} a+(1-\alpha) \sigma_{b} b}=\frac{\alpha \sigma_{g} c}{\alpha \sigma_{g} c+(1-\alpha) \sigma_{b} d}=\frac{1}{2}$. From these equalities we derive mixing probabilities on behalf of merger types: $\sigma_{b}=\frac{1-\frac{1-\alpha}{\alpha} \frac{c}{d}}{\frac{a d-c b}{a d}}$ and $\sigma_{g}=\frac{b d((1-\alpha) d-\alpha c)}{\alpha(a d-c b)}$. In order both mixing probabilities to be non-negative, condition $a d-c b>0$ should hold.

Mergers can appear in both decision nodes if they mix High and Low effort levels, i.e. both effort levels give the same payoffs to both types:

$a p_{H Y} \Pi_{g}-C(H)=c p_{L Y} \Pi_{g}-C(L)$

$b p_{H Y} \Pi_{b}-C(H)=d p_{L Y} \Pi_{b}-C(L)$

From these conditions we can derive optimal approval probabilities on behalf of the agency: $p_{L Y}=\frac{a \frac{C(H)-C(L)}{\Pi_{b}}-b \frac{C(H)-C(L)}{\Pi_{g}}}{b c-a d}$. In order for this probability to be non-negative condition $a d-c b<0$, which contradicts the previous condition.

Analogously $\mu_{H N} \neq \mu_{L N}$.

\section{Mixing equilibria}

ME1: $\bar{p}=\left(0, p_{H Y}, 0,0,0\right), \sigma_{g}=(1,0,0), \sigma_{b}=\left(\sigma_{b}^{H}, 0,\left[1-\sigma_{b}^{H}\right]\right), E T M=\alpha$ where $p_{H Y}=\frac{C(H)}{b \Pi_{b}}, \sigma_{b}^{H}=\frac{\alpha}{(1-\alpha)} \frac{a}{b} \in[0,1]$ for $\alpha \in\left[0, \frac{b}{a+b}\right]$

ME2: $\bar{p}=\left(0,0,0,0, p_{L Y}\right), \sigma_{g}=(0,1,0), \sigma_{b}=\left(0, \sigma_{b}^{L},\left[1-\sigma_{b}^{L}\right]\right), E T M=\alpha$ where $p_{L Y}=\frac{C(L)}{d \Pi_{b}}, \sigma_{b}^{L}=\frac{\alpha}{(1-\alpha)} \frac{c}{d} \in[0,1]$ for $\alpha \in\left[0, \frac{d}{c+d}\right]$

ME3: $\bar{p}=\left(0,1,1, p_{L N}, 1\right), \sigma_{g}=\left(\sigma_{g}^{H},\left[1-\sigma_{g}^{H}\right], 0\right), \sigma_{b}=(0,1,0), \operatorname{ETM}=1-\alpha$ where $p_{L N}=\frac{1}{1-c}\left[1-c-\frac{C(H)-C(L)}{\Pi_{g}}\right], p_{L N}: \Pi_{b}-C(H)<\left[p_{L N}(1-d)+d\right] \Pi_{b}-C(L)$ $\sigma_{g}^{H}=1-\frac{1-\alpha}{\alpha} \frac{1-d}{1-c} \in[0,1]$ for $\alpha \in\left[\frac{1-d}{2-c-d}, 1\right]$ 
ME4: $\bar{p}=\left(0,1, p_{H Y}, 0, p_{L Y}\right), \sigma_{g}=\left(\sigma_{g}^{H},\left[1-\sigma_{g}^{H}\right], 0\right), \sigma_{b}=\left(\sigma_{b}^{H},\left[1-\sigma_{b}^{H}\right], 0\right), E T M=$ $\alpha(1-a)+(1-\alpha) b$.

$\Pi_{g}\left(a+(1-a) p_{H N}^{M E 4}\right)-C(H)=\Pi_{g} c p_{L Y}^{M E 4}-C(L)$

$\Pi_{b}\left(b+(1-b) p_{H N}^{M E 4}\right)-C(H)=\Pi_{b} d p_{L Y}^{M E 4}-C(L)$

$\sigma_{b}^{H}=\frac{\alpha(1-a) c-(1-\alpha)(1-a) d}{(1-\alpha)(1-b) c-(1-\alpha)(1-a) d} \in[0,1]$

ME5: $\bar{p}=\left(0,1,1,0, p_{L Y}\right), \sigma_{g}=\left(\sigma_{g}^{H},\left[1-\sigma_{g}^{H}\right], 0\right), \sigma_{b}=(0,1,0), \operatorname{ETM}=(1-\alpha) \frac{d}{c}$.

$p_{L Y}^{M E 5}=\frac{1}{c}\left(1-\frac{C(H)-C(L)}{\Pi_{g}}\right) \in[0,1]$ and $\Pi_{b}-C(H)<p_{L Y}^{M E 5} d \Pi_{b}-C(L)$

$\sigma_{g}^{H}=1-\frac{1-\alpha}{\alpha} \frac{d}{c} \in[0,1]$ for $\alpha \in\left[\frac{d}{c+d}, 1\right]$

\section{Proposition 2:}

Equilibrium strategy ME5 $=\left(0110 p_{L Y}\right)$ gives lower expected mistake than:

a) E1 on the interval $\left(\frac{d-c b}{d-c b+c(1-a)} ; 1\right)$ if $\frac{a}{c}>\frac{b}{d}$;

b) E2 on the interval $\left(\frac{d}{c+d} ; 1\right)$ if $\frac{a}{c}<\frac{b}{d}$.

Proof: We compare expected mistakes under strategy $\left(0110 p_{L Y}\right)$ with all optimal equilibrium strategies E1 and E2: (00000), (00001), (01000), (11111).

Expected mistake for equilibrium strategy ME5 $=\left(0110 p_{L Y}\right)$ is $(1-\alpha) \frac{d}{c}$.

It is always true that $\left(0110 p_{L Y}\right) \succ(1111)$, because $(1-\alpha) \frac{d}{c}<(1-\alpha)$.

1) $\left(0110 p_{L Y}\right) \succ(00000)$, i.e. $(1-\alpha) \frac{d}{c}<\alpha$, if $\alpha>\frac{d}{c+d}$.

2) $\left(0110 p_{L Y}\right) \succ(01000)$, i.e. $(1-\alpha) \frac{d}{c}<\alpha(1-a)+(1-\alpha) b$, if $\alpha>\frac{d-c b}{d-c b+c(1-a)} \geq 0$.

3) $(01000) \succ(00000)$, i.e. $\alpha(1-a)+(1-\alpha) b<\alpha$, if $\alpha>\frac{b}{a+b}$.

4) $\left(0110 p_{L Y}\right) \succ(00001)$, i.e. $(1-\alpha) \frac{d}{c}<\alpha(1-c)+(1-\alpha) d$, if $\alpha>\frac{d}{c+d}$.

Part (a): It is always true that $\frac{d-c b}{d-c b+c(1-a)}>\frac{b}{a+b}$ if $\frac{a}{c}>\frac{b}{d}$. Hence, ME5 has a lower expected mistake than E1 if $\alpha \in\left(\frac{d-c b}{d-c b+c(1-a)} ; 1\right)$.

Part (b): If $\frac{a}{c}<\frac{b}{d}$, then $\left(0110 p_{L Y}\right) \succ(00000)$ and $\left(0110 p_{L Y}\right) \succ(00001)$ for $\alpha>\frac{d}{c+d}$. Hence, ME5 has a lower mistake than equilibrium E2 if $\alpha \in\left(\frac{d}{c+d} ; 1\right)$.

\section{Full commitment equilibrium:}

a) $\alpha \in\left[0, \alpha_{1}\right), p=\left(0, p_{H Y}^{F C}, 0,0,0\right), \sigma_{g}=(1,0,0), \sigma_{b}=(0,0,1)$,

$E T M=\alpha-\alpha$ a $p_{H Y}^{F C}$

b) $\alpha \in\left[\alpha_{1}, \alpha_{2}\right), p=\left(0,1,0,0, p_{L Y}^{F C}\right), \sigma_{g}=(1,0,0), \sigma_{b}=(0,1,0)$,

$E T M=\alpha(1-a)+(1-\alpha) d p_{L Y}^{F C}$

c) $\alpha \in\left[\alpha_{2}, 1\right], p=\left(0,1,1, p_{L N}^{F C}, 1\right), \sigma_{g}=(1,0,0), \sigma_{b}=(0,1,0)$,

$E T M=(1-\alpha) d+(1-\alpha)(1-d) p_{L N}^{F C}$

where $\alpha_{1}=\frac{b-d-\frac{C(H)-C(L)}{\Pi_{b}}}{a\left(1-\frac{C(H)}{b \Pi_{b}}\right)+\left(b-d-\frac{C(H)-C(L)}{\Pi_{b}}\right)}, \alpha_{2}=\frac{1-b}{2-a-b}$,

$p_{H Y}^{F C}=\frac{C(H)}{b \Pi_{b}}-\varepsilon, \quad p_{L N}^{F C}=\frac{1}{1-d}\left[1-d-\frac{C(H)-C(L)}{\Pi_{b}}\right]+\varepsilon, \quad p_{L Y}^{F C}=\frac{1}{d}\left[b-\frac{C(H)-C(L)}{\Pi_{b}}\right]+\varepsilon$.

and $p_{L Y}^{F C}: a \Pi_{g}-C(H)>p_{L Y}^{F C} c \Pi_{b}-C(L), \varepsilon \rightarrow 0$. 


\section{Footnote 20:}

If $\frac{C(H)}{C(L)}>\frac{c b}{a d}$ and $p_{H Y}=\frac{C(H)}{b \Pi_{b}}-\varepsilon, p_{L Y}=\frac{C(L)}{d \Pi_{b}}-\varepsilon$, then " $\left(0 p_{H Y} 000\right) " \succ$ " $\left(0000 p_{L Y}\right) "$.

Proof: Expected mistakes: $\alpha a\left(1-p_{H Y}\right)+\alpha(1-a)<\alpha c\left(1-p_{L Y}\right)+\alpha(1-c) \Leftrightarrow$ $\alpha a\left(1-\frac{C(H)}{b \Pi_{b}}\right)+\alpha(1-a)<\alpha c\left(1-\frac{C(L)}{d \Pi_{b}}\right)+\alpha(1-c) \Leftrightarrow \frac{C(H)}{C(L)}>\frac{c b}{a d}$ (if $\frac{a}{c}>\frac{b}{d}$, then it is always true).

\section{Proposition 3:}

If the commitment to reject all mergers that have not produced the evidence is credible and $\frac{a}{c}<\frac{b}{d}$, then equilibrium strategy $P C E=\left(0100 p_{L Y}^{P C}\right)$ gives a smaller expected total mistake than E2 on the interval $\alpha \in\left(\frac{d}{c+d} ; \frac{1-a \frac{d}{c}}{2-a-a \frac{d}{c}}\right)$.

Proof: We compare expected mistakes under strategy $\left(0100 p_{L Y}^{P C}\right)$ with all equilibrium strategies under E2: (00000), (00001), (01000),(11111).

To have $\left(0100 p_{L Y}^{P C}\right)$ as an equilibrium strategy, the merging firms choose $\sigma_{g}=\left(\sigma_{g}^{H},[1-\right.$ $\left.\left.\sigma_{g}^{H}\right], 0\right), \sigma_{b}=(0,1,0)$, where $\sigma_{g}^{H}=\left[1-\frac{(1-\alpha)}{\alpha} \frac{d}{c}\right]$ to make the agency indifferent between approving or rejecting a merger and $p_{L Y}^{P C}=\frac{1}{c}\left(a-\frac{C(H)-C(L)}{\Pi_{g}}\right)$ to make a 'good' merger indifferent between High and Low effort levels and 'bad' merger chooses Low effort level.

Strategy $\left(0100 p_{L Y}^{P C}\right)$ gives the following expected total mistake:

$\alpha \sigma_{g}^{H}(1-a)+\alpha\left(1-\sigma_{g}^{H}\right)\left(1-c p_{L Y}^{P C}\right)+(1-\alpha) d p_{L Y}^{P C}$.

The first term in this expression comes from the fact that the agency commits to reject all mergers that have not produced the evidence, even though there are only 'good' mergers in $\left(e^{H}, N o\right)$ decision node. After plugging in the formulas for $\sigma_{g}^{H}$ and $p_{L Y}^{P C}$, the expected total mistake is: $\alpha(1-a)+(1-\alpha) a \frac{d}{c}$. Hence,

1. Strategy $\left(0100 p_{L Y}^{P C}\right) \succ(01000)$, if

$$
\alpha(1-a)+(1-\alpha) a \frac{d}{c}<\alpha(1-a)+(1-\alpha)(1-b) \quad \Leftrightarrow \quad \frac{a}{c}<\frac{b}{d}
$$

2. Strategy $\left(0100 p_{L Y}^{P C}\right) \succ(11111)$, if $\alpha(1-a)+(1-\alpha) a \frac{d}{c}<1-\alpha \Leftrightarrow \alpha<\frac{1-a \frac{d}{c}}{2-a-a \frac{d}{c}}$

3. Strategy $\left(0100 p_{L Y}^{P C}\right) \succ(00000)$, if $\alpha(1-a)+(1-\alpha) a \frac{d}{c}<\alpha \Leftrightarrow \alpha>\frac{d}{c+d}$

4. Strategy $\left(0100 p_{L Y}^{P C}\right) \succ(00001)$, if

$$
\alpha(1-a)+(1-\alpha) a \frac{d}{c}<\alpha(1-c)+(1-\alpha) d \quad \Leftrightarrow \quad \alpha>\frac{d}{c+d} .
$$

Thus strategy $P C E \succ E 2$ on the interval $\alpha \in\left(\frac{d}{c+d} ; \frac{1-a \frac{d}{c}}{2-a-a \frac{d}{c}}\right)$ when $\frac{a}{c}<\frac{b}{d}$. 


\section{Appendix B:}

Diagram 1. Extensive form of the game
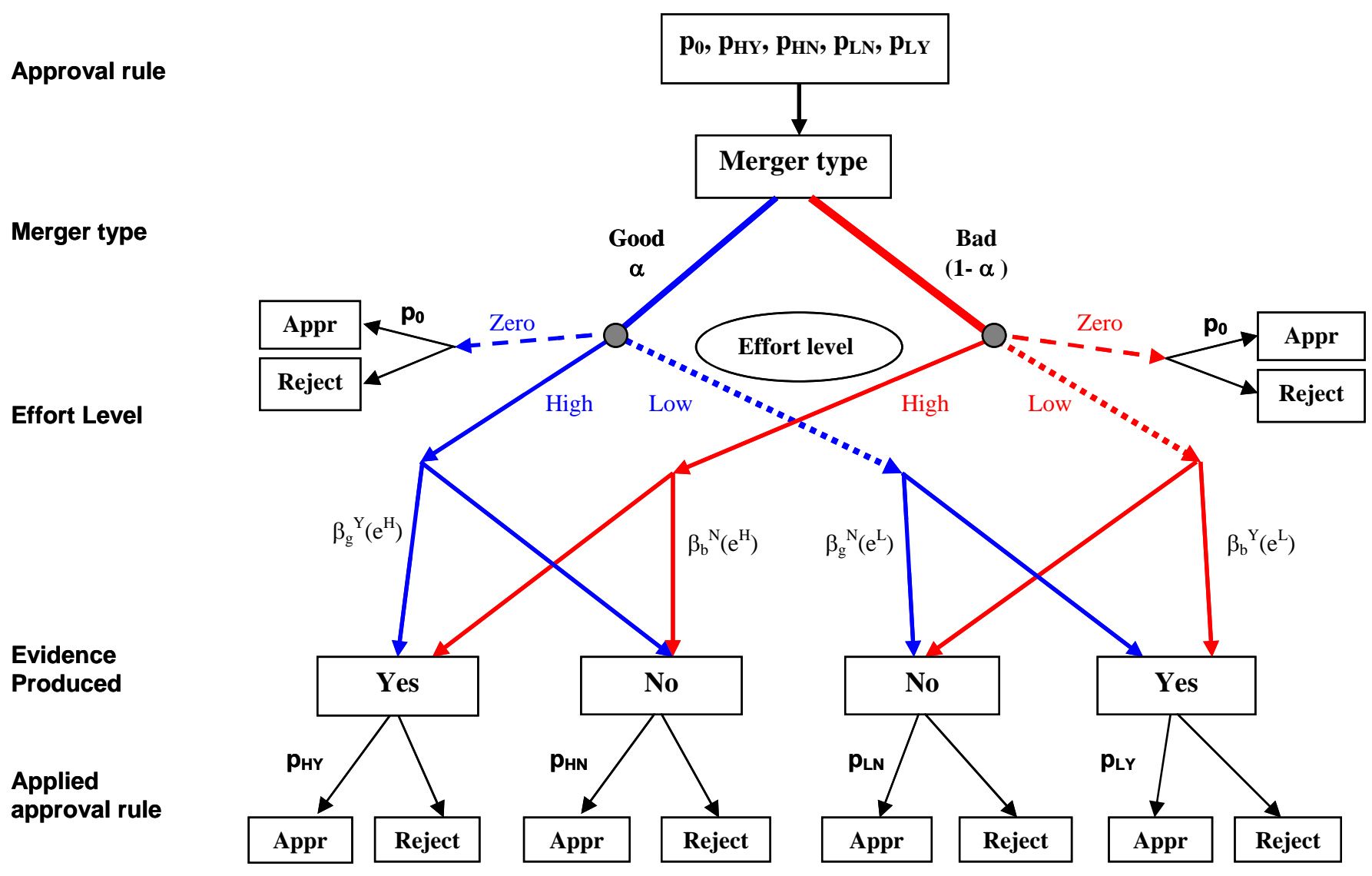

Evidence

Produced

Applied

approval rule

Effort Level

Agency's Payoff is the summation of Type I and Type II errors 
Appendix C:
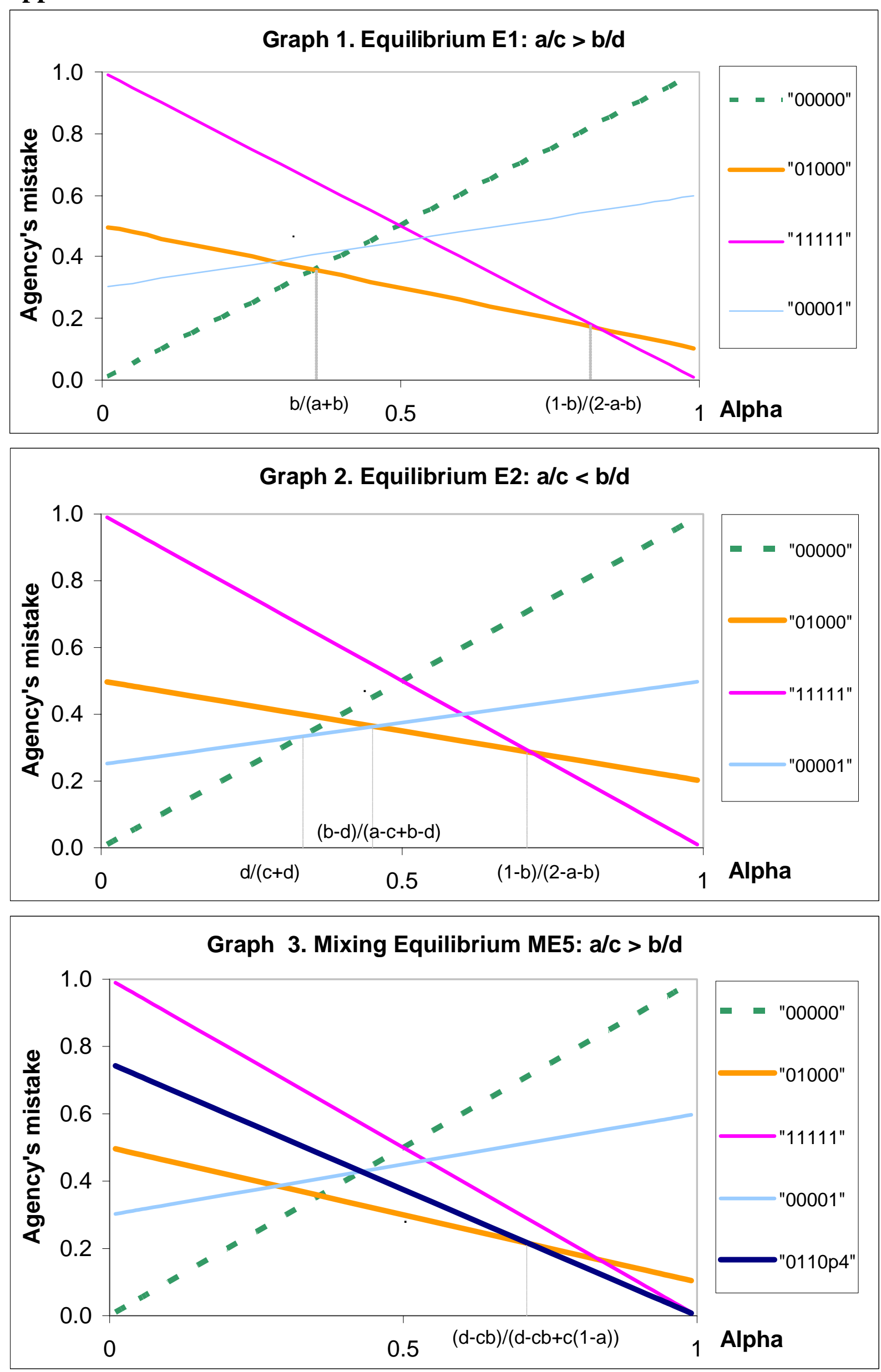

26 

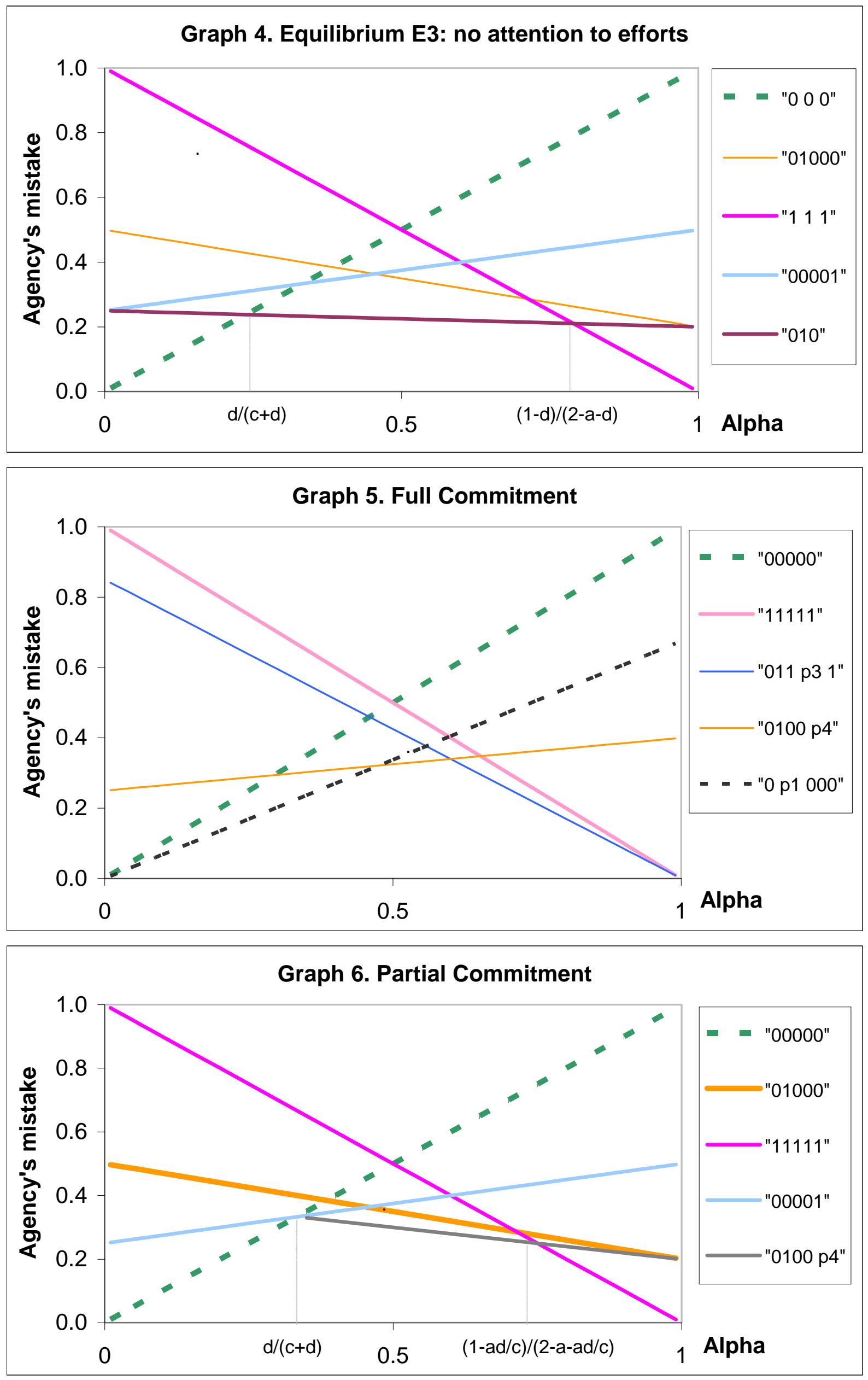

27 